\title{
Congenital dyserythropoietic anemia type II (CDA II) diagnosed in an adult patient
}

\author{
Bevan Tandon • LoAnn C. Peterson • \\ Stephanie Norwood • Anaadriana Zakarija • \\ Yi-Hua Chen
}

Received: 22 October 2010 / Accepted: 3 November 2010 / Published online: 19 November 2010

(C) Springer-Verlag 2010

\begin{abstract}
The congenital dyserythropoietic anemias (CDAs) are a group of rare hereditary disorders characterized by ineffective erythropoiesis and distinct morphologic abnormalities of the erythroblasts in the bone marrow. CDA type II (CDA II) is the most common subtype, with more than 300 cases reported in the literature. Patients often present with anemia, jaundice, and variable splenomegaly. Most cases of CDA II are diagnosed at a young age; some patients, however, may remain unrecognized until late adulthood. Unless CDA is considered in the differential diagnosis for anemia, adult patients are often subjected to extensive clinical work-up for many years prior to accurate diagnosis. Early recognition of CDA II is important to prevent end-organ damage secondary to iron overload, the most important complication of the disease. We report a case of CDA II diagnosed in a 48 -yearold woman with a longstanding history of anemia of unknown etiology.
\end{abstract}

Keywords Congenital dyserythropoietic anemia type II (CDA II) · Hereditary erythroblastic multinuclearity with a positive acidified serum test (HEMPAS) - Anemia . Dyserythropoiesis $\cdot$ Ineffective erythropoiesis

\footnotetext{
B. Tandon · L. C. Peterson · S. Norwood · Y.-H. Chen $(\bowtie)$ Department of Pathology, Northwestern University Feinberg School of Medicine,

251 E. Huron, Feinberg 7-209A,

Chicago, IL 60611, USA

e-mail: y-chen5@northwestern.edu
}

\section{A. Zakarija}

Division of Hematology/Oncology, Department of Internal Medicine, Northwestern University Feinberg School of Medicine, Chicago, IL, USA

\section{Introduction}

The congenital dyserythropoietic anemias (CDAs) are a group of rare hereditary anemias resulting from constitutional defects in erythrocyte maturation associated with characteristic dysplastic features involving bone marrow erythroblasts and were initially described by Wolff and von Hofe in 1951 [1]. The classification scheme originally outlined by Heimpel and Wendt in 1968 is still in use today, subcategorizing the CDAs as Type I, Type II, and Type III $[2,3]$. Various proposed new subtypes have also been published [4-6].

CDA II is the most common subtype of CDA with more than 300 cases reported in the literature, predominantly within European and Mediterranean populations. The severity of anemia in CDA II ranges from mild to transfusion-dependent. Other clinical features include splenomegaly resulting from sequestration of abnormal circulating erythrocytes and jaundice due to unconjugated hyperbilirubinemia. Secondary hemochromatosis from iron overload may also occur in some patients especially if left untreated [7].

CDA II exhibits autosomal recessive transmission. Since its inception, the molecular pathogenesis of CDA II was not fully defined and was thought to be owed predominantly to defects in glycosylation of the red cell membrane proteins band 3 (anion exchange protein transporter 1) and band 4.5 (glucose transporter 1) [8, 9]. Genome-wide linkage analysis relegated genetic mutations to the long arm of chromosome 20 in a region referred as the CDAN 2 locus [10]; however, the majority of specific candidate genes within this region had been excluded [11]. As recently as 2009, investigators have demonstrated that CDA II is caused by missense mutations in the SEC23B gene 
encoding coat protein complex II (COPII), complex proteins critical for membrane homeostasis and vesicular trafficking from the endoplasmic reticulum to the golgi complex in eukaryotes [12]. Disruption of SEC23B gene expression has been shown to recapitulate the nuclear cytokinesis and double cell membrane defects characteristic of CDA II erythroblasts [13, 14].

The current "gold standard" for diagnosis of CDA II is bone marrow biopsy in conjunction with laboratory tests. We present a case of CDA II diagnosed in a 48-year-old woman who presented with longstanding anemia of unknown etiology.

\section{Case report}

The patient is a 48-year-old woman of Greek descent referred to our hospital for management of fatigue and longstanding anemia of unknown etiology since childhood. The patient had been subjected to an extensive clinical work-up for many years including hemoglobin electrophoresis, serum iron studies, folate and B12 levels, antibody screening, whole blood lead levels, and thyroid and liver function tests, the results of which were all within normal ranges. Complete blood count at presentation showed: WBC, $8.2 \mathrm{k} / \mu \mathrm{L} ; \mathrm{RBC}, 3.46 \mathrm{M} / \mu \mathrm{L}$; Hb, $9.8 \mathrm{~g} / \mathrm{dL}$; HCT, 28.4\%; MCV, $82 \mathrm{fL}$; MCH, 28.2 pg; MCHC, $34.4 \mathrm{~g} / \mathrm{dL}$; RDW, 16.4\%; and platelet, $228 \mathrm{k} / \mu \mathrm{L}$. Absolute reticulocyte count was normal $(0.1166 \mathrm{M} / \mu \mathrm{L})$. Physical examination revealed mild scleral icterus; no splenomegaly or other abnormal findings were noted. Iron studies showed elevated ferritin $(598.3 \mathrm{ng} / \mathrm{mL})$, normal serum iron $(122 \mathrm{~g} / \mathrm{dL})$, and normal total iron binding capacity $(264 \mu \mathrm{g} / \mathrm{dL})$. Total bilirubin was elevated $(2.3 \mathrm{~g} / \mathrm{dL})$, and direct bilirubin was within normal limits $(0.2 \mathrm{~g} / \mathrm{dL})$. Serum haptoglobin level was decreased $(28 \mathrm{mg} / \mathrm{dL})$. No abnormal hemoglobin was detected by hemoglobin electrophoresis. Review of the peripheral blood smear showed normocytic normochromic anemia with occasional teardrop forms and nucleated red blood cells, and rare myelocytes were also present. Therefore, a bone marrow biopsy was performed to rule out an infiltrative process in the bone marrow.

\section{Results}

The peripheral blood smears showed a normochromic, normocytic anemia with increased polychromasia and anisopoikilocytosis including occasional teardrop forms. Occasional red blood cells with coarse basophilic stippling were seen. Occasional circulating nucleated red blood cells were also present, including binucleated forms (Fig. 1) Neutrophils were adequate with unremarkable morphology;

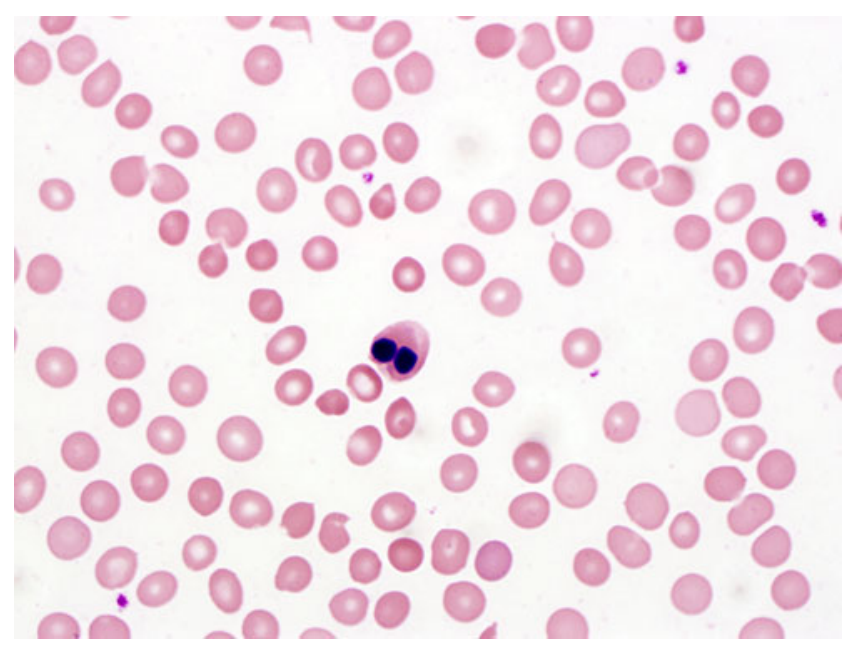

Fig. 1 Peripheral blood smear showed normochromic, normocytic anemia with increased polychromasia and anisopoikilocytosis including occasional teardrop forms. Occasional nucleated red blood cells were also present, including binucleated forms $(\times 1000$, WrightGiemsa stain)

rare myelocytes were present. Platelets were adequate in number with unremarkable morphology.

The bone marrow aspirate smears demonstrated marked proliferation of erythroid precursors with a myeloid to erythroid ratio of $1: 10$. The most striking feature was the prominent binucleation of the erythroblasts characterized by equal size of the two nuclei; occasional multinucleated erythroblasts were also seen (Figs. 2 and 3). These binucleated erythroblasts represented approximately $40 \%$ of the total erythroid precursors, and were all at the later stages of maturation, mostly late polychromatophilic or orthochromatophilic erythroblasts. Myeloid precursors showed normal, progressive maturation, and megakaryocytes were morphologically unremarkable. Sections of the bone marrow core biopsy displayed marked hypercellularity ( $95 \%$ cellular) with erythroid predominance. The binucleation of the erythroid precursors was also easily appreciated in the core biopsy (Fig. 4). Results of flow cytometric immunophenotyping of the bone marrow aspirate were unremarkable. Cytogenetic analysis revealed a normal female karyotype.

A diagnosis of CDA II was made based on the characteristic bone marrow findings of binucleation with equal-sized nuclei in approximately $40 \%$ of late polychromatophilic or orthochromatophilic erythroblasts in conjunction with the clinical history of longstanding anemia since childhood, clinical and laboratory evidence of hemolysis (scleral icterus, unconjugated hyperbilirubinemia, and decreased haptoglobin), and evidence of ineffective erythropoiesis reflected by the normal absolute reticulocyte count in the peripheral blood in the presence of marked erythroid hyperplasia in the bone marrow. 


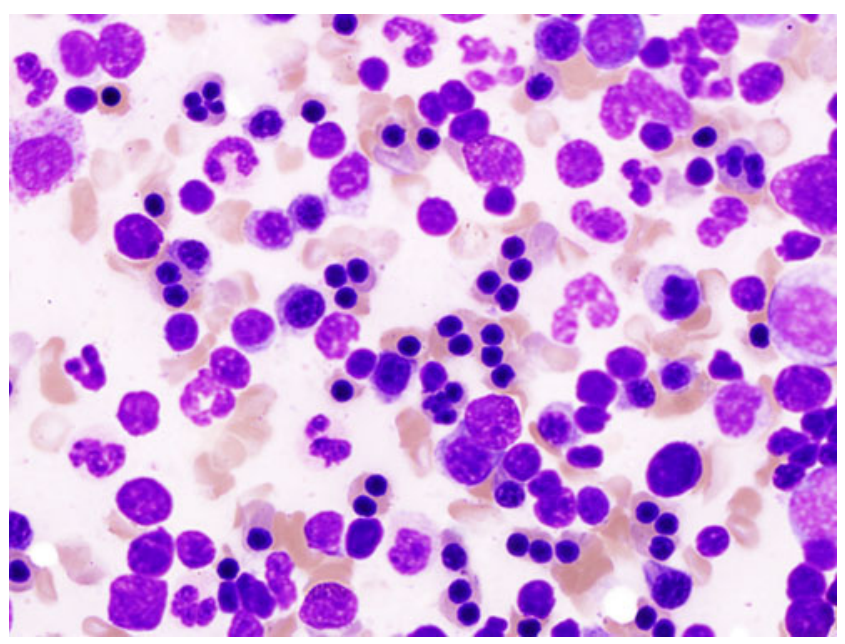

Fig. 2 Bone marrow aspirate smear revealed erythroid hyperplasia and frequent binucleation in erythroblasts $(\times 1000$, Wright-Giemsa stain)

The patient's management has included folic acid supplementation, monitoring of serum ferritin levels every 3 months, and recommendations for splenectomy in the event of progression in severity of anemia or iron chelation therapy should ferretin levels exceed $1,000 \mathrm{ng} / \mathrm{mL}$. During the course of 3 years of clinical follow-up, the patient's performance status and severity of anemia have shown no significant changes, but her serum ferritin level has been increased up to $744.9 \mathrm{ng} / \mathrm{mL}$ (normal reference ranges, $11.0-307.0 \mathrm{ng} / \mathrm{mL}$ ).

\section{Discussion}

CDA II is also known as hereditary erythroblastic multinuclearity with a positive acidified serum test (HEMPAS),

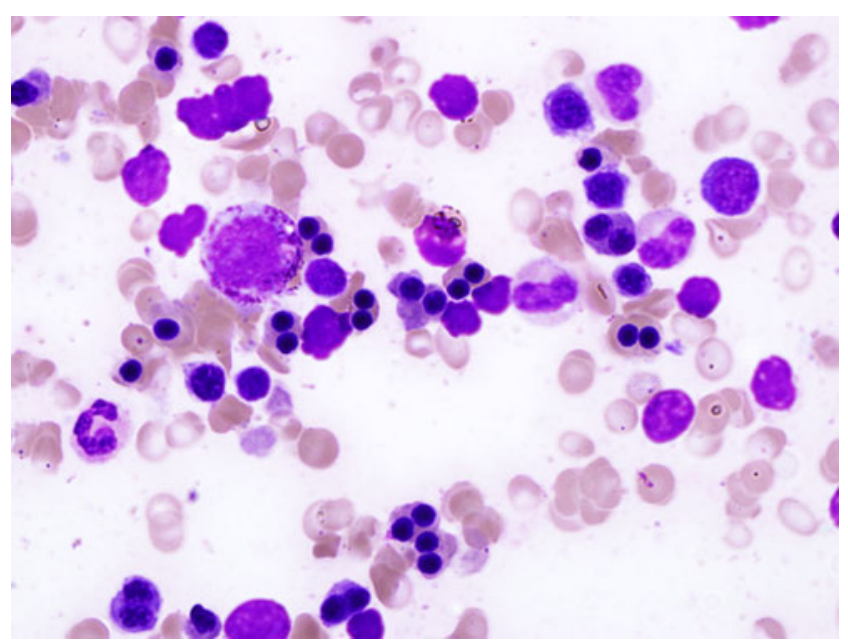

Fig. 3 The binucleation of erythroblasts was characterized by two equal-sized nuclei and present in the erythroblasts at the later stages of maturation, mostly late polychromatophilic or orthochromatophilic erythroblasts $(\times 1000$, Wright-Giemsa stain $)$

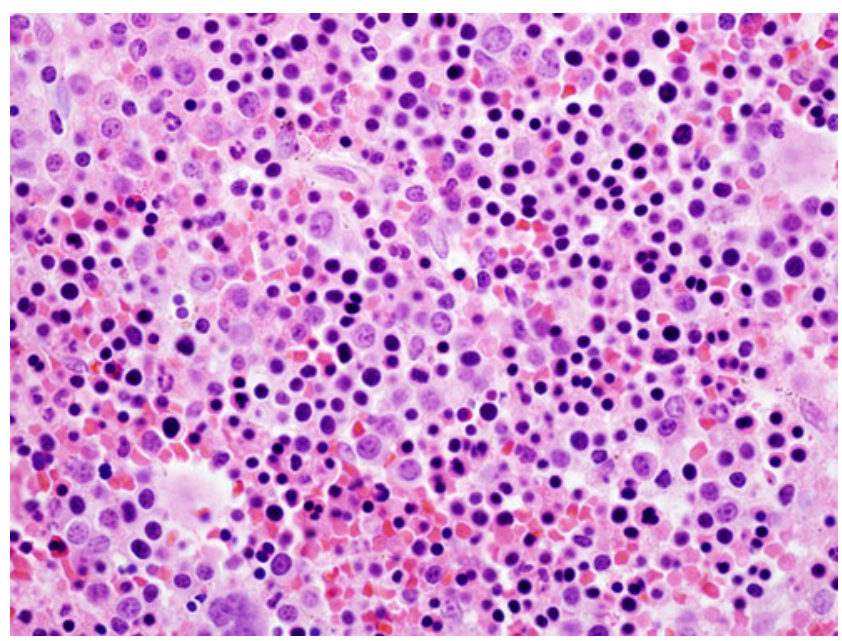

Fig. 4 Bone marrow core biopsy demonstrated marked erythroid hyperplasia. The binucleation of erythroblasts were readily appreciated $(\times 600, H \&$ E stain $)$

familial benign erythroblastic polyploidy, and hemolytic splenomegalic erythropolydyskaryosis. It is the most widely documented subtype of CDA, with approximately 367 cases reported in the literature, predominantly in European populations [15]. The term HEMPAS derives from observations by Crookston et al. that the red blood cells from CDA II patients demonstrated susceptibility to lysis in ABO-compatible normal sera in the acidified serum lysis test (HAM's test) [16]. CDA II is an autosomal recessive disorder. The anemia in CDA II was initially thought to be due to ineffective erythropoiesis secondary to the glycosylation defects of red blood cell membrane proteins. Recent studies have demonstrated that missense mutations in SEC23B gene are responsible for the disease. These mutations result in the impaired expression of COPII proteins which are essential for functional golgi processing in erythrocytes.

Although many CDA II patients are diagnosed in their infancy or childhood, some patients, as in our case, remain unrecognized until late adulthood likely secondary to less severe anemia. Patients often have splenomegaly and jaundice, and less frequently, hepatomegaly. Our patient had scleral icterus at presentation, but no organomegaly was identified. Transfusion dependence is seen in approximately in $10 \%$ of CDA II patients during infancy and early childhood, but usually resolves with age. Although typically considered a relatively benign condition, neonatal mortality has been documented, likely secondary to inadequate functional marrow reserves during early life [17]. Cholelithiasis may be seen with increased frequency in patients concurrently suffering from Gilbert's syndrome [18]. Rare instances of extramedullary hematopoiesis-related paravertebral tumors have been reported, as have associations with various hematological and non-hematological disorders 
including Dubin-Johnson syndrome, Von Willebrand's disease, mental retardation, and Sweet's syndrome [19-21].

As exemplified in our case, CDA II patients may have a relatively indolent clinical course due to less severe hemolytic anemia, thus resulting in delayed diagnosis. When patients present in late adulthood for evaluation of anemia, CDA becomes even less of a differential diagnostic consideration, and therefore, a bone marrow biopsy may not be performed. In addition, many pathologists may not be familiar with the typical bone marrow morphology in CDA II patients. The morphologic findings of the peripheral blood in CDA II patients are often highly nonspecific. In our case, coarse basophilic stippling was noted in erythrocytes which may be seen in lead intoxication, megaloblastic anemia, thalessemia, or other severe anemias. The presence of occasional teardrop red blood cells, nucleated red blood cells, and rare myelocytes in the peripheral blood smears in our case prompted bone marrow biopsy to rule out a marrow infiltrative process. Bone marrow examination remains the "gold standard" for diagnosis of CDA II. The characteristic feature in the bone marrow in CDA II is erythroblastic binucleation with equal size of the two nuclei [22]. The binucleation is usually present in more than $10 \%$ of erythroblasts, and is typically restricted to late polychromatophilic or orthochromatophilic erythroblasts. Karyorrhexis is common, and multinucleation may also be present. Pseudo-Gaucher cells may be seen, reflecting phagocytosis of erythropoietic debris from ineffective erythropoiesis within the reticuloendothelial system [23].

Diagnosis of CDA II in our case was based on the characteristic bone marrow findings of binucleation with equal-sized nuclei in approximately $40 \%$ of late polychromatophilic or orthochromatophilic erythroblasts, clinical history of longstanding anemia since childhood, and clinical and laboratory evidence of hemolysis and ineffective erythropoiesis.

According to previously proposed classification schemes [24, 25], confirmation of CDA II includes sodium dodecyl sulfate polyacrylamide gel electrophoresis for erythrocyte band 3 protein [26], acidified serum lysis testing (HAM's test) [16], electron microscopy to demonstrate the presence of a double membrane adjacent the cell membrane in mature red blood cells [27], or assessment of mutation status of the recently recognized SEC3B gene [12]. These specialized tests, however, are not available in most clinical laboratories. Heimpel et al. have recently evaluated the morphologic findings of the peripheral blood and bone marrow biopsies from 36 confirmed CDA II cases obtained from the German Registry of Congenital Dyserythropoietic Anemias and found that if more than $10 \%$ of typical binucleated erythroblasts are seen, together with more than $2 \%$ of cells with karyorrhexis, the diagnosis of CDA II is essentially confirmed [23]. In our patient, approximately
$40 \%$ of mature erythroblasts showed binucleation with two equal-sized nuclei, characteristic for CDA II.

The most important complication of CDA II is iron overload, reflected by an increased ferritin level, with kinetics similar to untreated hereditary hemochromatosis or thalassemia intermedia. Serum ferritin levels increase with time in both untransfused and transfused patients, irrespective of the HFE H63D genotype, suggesting that increased erythropoiesis is the primary cause of iron overload [28]. Although the precise mechanism responsible for iron overload in CDA II has not been elucidated, secondary hemochromatosis in CDA II patients likely stems from increased iron absorption in response to ineffective erythropoiesis and secondary erythroid hyperplasia. Severe endorgan damage has been noted to occur in CDA II patients not receiving treatment for iron overload. Indeed, congestive heart failure from CDA II-related secondary hemochromatosis has been documented [17]. Iron chelation therapy represents the mainstay of supportive management of CDA II patients. Therefore, early recognition of CDA II is important in preventing end-organ damage secondary to iron overload [29]. Since the diagnosis of CDA II was made on the bone marrow biopsy, our patient has been followed up for ferritin levels every 3 months. She has shown elevations in serum ferritin levels up to $744.9 \mathrm{ng} / \mathrm{mL}$ during the course of 3 years of follow-up. It has been recommended that iron chelation therapy will be administered when the ferretin level exceeds $1,000 \mathrm{ng} / \mathrm{mL}$.

Clinically, CDA II is not infrequently misdiagnosed as hereditary spherocytosis (HS) due to hemolysis-related symptoms and laboratory findings. CDA II, however, may exhibit reticulocytosis incommensurate to the degree of anemia as compared to HS which typically shows an adequate marrow compensatory response. Of note, our patient showed an absolute reticulocyte count within normal range despite marked erythroid hyperplasia in the bone marrow, reflecting the ineffective erythropoiesis in CDA II patients. Dyserythropoiesis can be seen in various conditions including exposure to heavy metals, particularly arsenic, and certain medications, vitamin B12 and folic acid deficiency, and myelodysplastic syndrome (MDS). Erythroblasts in these conditions often show a wide range of dysplastic features including megaloblastic changes, karyorrhexis, nuclear budding, internuclear bridging, or multinucleation. Binucleation with equal-sized nuclei characteristic of CDA II, however, is never the predominant feature in these conditions. Additional clinical, laboratory, and ancillary studies, as well as morphologic findings in other cell lineages in the bone marrow, assist in the differential diagnosis. Dysplastic features in CDA I and CDA III are characterized by internuclear bridging and giant erythroblasts with highly complex nuclei, respectively. These features may also be seen in other conditions such as arsenic poisoning or MDS. 
Therefore, CDA should be considered as a cause of dysplasia when it is confined to the erythroblasts [30].

In summary, CDA II represents the most common subtype of the CDAs. Patients may remain undiagnosed until well into adulthood due to the variability in the severity of anemia and the nonspecific morphologic findings in the peripheral blood. Bone marrow biopsy is the "gold standard" for diagnosis of CDA II, and is characterized by erythroid hyperplasia and binucleation of equal-sized nuclei in at least $10 \%$ of more mature erythroblasts. Despite the clinical picture of hemolytic anemia and marked erythroid hyperplasia in the bone marrow, the absolute reticulocyte count is often normal, reflecting the ineffective erythropoiesis in CDA patients. It is important to include CDAs in the differential diagnosis in adult patients with longstanding anemia and dysplasia in the bone marrow confined to the erythroblasts. Early recognition of CDA II is important to prevent end-organ damage secondary to iron overload.

Conflict of interest The authors have no conflict of interest.

\section{References}

1. Wolff JA, Von Hofe FH (1951) Familial erythroid multinuclearity. Blood 6:1274-1283

2. Heimpel H, Wendt F (1968) Congenital dyserythropoietic anemia with karyorrhexis and multinuclearity of erythroblasts. Helv Med Acta 34:103-115

3. Heimpel H (2004) Congenital dyserythropoietic anemias: epidemiology, clinical significance, and progress in understanding their pathogenesis. Ann Hematol 83:613-621

4. Wickramasinghe SN, Spearing RL, Hill GR (1998) Congenital dyserythropoiesis with intererythroblastic chromatin bridges and ultrastructurally-normal erythroblast heterochromatin: a new disorder. Br J Haematol 103:831-834

5. Heimpel H, Kohne E, Schrod L, Schwarz K, Wickramasinghe S (2007) A new type of transfusion-dependent congenital dyserythropoietic anemia. Haematologica 92:1427-1428

6. Woessner S, Trujillo M, Florensa L, Mesa MC, Wickramasinghe SN (2003) Congenital dyserthropoietic anaemia other than type I to III with a peculiar erythroblastic morphology. Eur J Haematol 71:211-214

7. Cazzola M, Barosi G, Bergamaschi G, Dezza L, Palestra P et al (1983) Iron loading in congenital dyserythropoietic anaemias and congenital sideroblastic anaemias. Br J Haematol 54:649-654

8. Fukuda MN (1993) Congenital dyserythropoietic anaemia type II (HEMPAS) and its molecular basis. Baillières Clin Haematol 6:493-511

9. Fukuda MN (1990) HEMPAS disease: genetic defect of glycosylation. Glycobiology 1:9-15

10. Gasparini P, Miraglia del Giudice E, Delaunay J, Totaro A, Granatiero $M$ et al (1997) Localization of the congenital dyserythropoietic anemia II locus to chromosome $20 \mathrm{q} 11.2$ by genomewide search. Am J Hum Genet 61:1112-1116

11. Iolascon A, Miraglia del Giudice E, Perrotta S, Granatiero M, Zelante L et al (1997) Exclusion of three candidate genes as determinants of congenital dyserythropoietic anemia type II (CDA-II). Blood 90:4197-4200
12. Bianchi P, Fermo E, Vercellati C, Boschetti C, Barcellini W et al (2009) Congenital dyserythropoietic anemia type II (CDAII) is caused by mutations in the SEC23B gene. Hum Mutat 30:1292-1298

13. Schwarz K, Iolascon A, Verissimo F, Trede NS, Horsley W et al (2009) Mutations affecting the secretory COPII coat component SEC23B cause congenital dyserythropoietic anemia type II. Nat Genet 41:936-940

14. Iolascon A, Russo R, Esposito MR, Asci R, Piscopo C et al (2010) Molecular analysis of 42 patients with congenital dyserythropoietic anemia type II: new mutations in the SEC23B gene and a search for a genotype-phenotype relationship. Haematologica 95:708-715

15. Heimpel H, Matuschek A, Ahmed M, Bader-Meunier B, Colita A et al (2010) Frequency of congenital dyserythropoietic anemias in Europe. Eur J Haematol 85:20-25

16. Crookston JH, Crookston MC, Burnie KL, Francombe WH, Dacie JV et al (1969) Hereditary erythroblastic multinuclearity associated with a positive acidified-serum test: a type of congenital dyserythropoietic anaemia. Br J Haematol 17:11-26

17. Iolascon A, Delaunay J, Wickramasinghe SN, Perrotta S, Gigante $M$ et al (2001) Natural history of congenital dyserythropoietic anemia type II. Blood 98:1258-1260

18. Perrotta S, del Giudice EM, Carbone R, Servedio V, Schettini F Jr et al (2000) Gilbert's syndrome accounts for the phenotypic variability of congenital dyserythropoietic anemia type II (CDAII). J Pediatr 136:556-559

19. Clauvel JP, Erlinger S (1974) Congenital dyserythropoietic anemia and the Dubin-Johnson syndrome. A case report. Gastroenterology 67:686-690

20. Hernandez P, Almagro D, Corral JF, Opolski A, Sanchez JA et al (1974) Association of type II congenital dyserythropoietic anaemia and von Willebrand's disease. Br J Haematol 27:453-462

21. Majeed HA, Kalaawi M, Mohanty D, Teebi AS, Tunjekar MF et al (1989) Congenital dyserythropoietic anemia and chronic recurrent multifocal osteomyelitis in three related children and the association with Sweet syndrome in two siblings. J Pediatr 115:730-734

22. Queisser W, Spiertz E, Jost E, Heimpel H (1971) Proliferation disturbances of erythroblasts in congenital dyserythropoietic anemia type I and II. Acta Haematol 45:65-76

23. Heimpel H, Kellermann K, Neuschwander N, Hogel J, Schwarz K (2010) The morphological diagnosis of congenital dyserythropoietic anemia: results of a quantitative analysis of peripheral blood and bone marrow cells. Haematologica 95:1034-1036

24. Heimpel H, Schwarz K, Ebnother M, Goede JS, Heydrich D et al (2006) Congenital dyserythropoietic anemia type I (CDA I): molecular genetics, clinical appearance, and prognosis based on long-term observation. Blood 107:334-340

25. Heimpel H, Anselstetter V, Chrobak L, Denecke J, Einsiedler B et al (2003) Congenital dyserythropoietic anemia type II: epidemiology, clinical appearance, and prognosis based on long-term observation. Blood 102:4576-4581

26. Anselstetter V, Horstmann HJ, Heimpel H (1977) Congenital dyserythropoietic anaemia, types I and II: aberrant pattern of erythrocyte membrane proteins in CDA II, as revealed by two-dimensional polyacrylamide gel electrophoresis. Br J Haematol 35:209-215

27. Heimpel H, Forteza-Vila J, Queisser W, Spiertz E (1971) Electron and light microscopic study of the erythroblasts of patients with congenital dyserythropoietic anemia. Blood 37:299-310

28. Van Steenbergen W, Matthijs G, Roskams T, Fevery J (2002) Noniatrogenic haemochromatosis in congenital dyserythropoietic anaemia type II is not related to $\mathrm{C} 282 \mathrm{Y}$ and $\mathrm{H} 63 \mathrm{D}$ mutations in the HFE gene: report on two brothers. Acta Clin Belg 57:79-84

29. Halpern Z, Rahmani R, Levo Y (1985) Severe hemochromatosis: the predominant clinical manifestation of congenital dyserythropoietic anemia type 2. Acta Haematol 74:178-180

30. Swerdlow S, Campo E, Harris N et al (2008) WHO. Classification of tumours of haematopoietic and lymphoid tissues, 4th ed. 88-91. 